Draft Version November 23, 2018

Preprint typeset using $\mathrm{LATE}_{\mathrm{E}} \mathrm{X}$ style emulateapj v. 03/07/07

\title{
A POSSIBLE EVOLUTIONARY SCENARIO OF HIGHLY MAGNETIZED SUPER-CHANDRASEKHAR WHITE DWARFS: PROGENITORS OF PECULIAR TYPE IA SUPERNOVAE
}

\author{
Upasana Das ${ }^{1}$, Banibrata Mukhopadhyay ${ }^{1}$, A. R. RaO ${ }^{2}$ \\ Draft version November 23, 2018
}

\begin{abstract}
Several recently discovered peculiar type Ia supernovae seem to demand an altogether new formation theory that might help explain the puzzling dissimilarities between them and the standard type Ia supernovae. The most striking aspect of the observational analysis is the necessity of invoking superChandrasekhar white dwarfs having masses $\sim 2.1-2.8 M_{\odot}, M_{\odot}$ being the mass of Sun, as their most probable progenitors. Strongly magnetized white dwarfs having super-Chandrasekhar masses were already established to be potential candidates for the progenitors of peculiar type Ia supernovae. Owing to the Landau quantization of the underlying electron degenerate gas, theoretical results yielded the observationally inferred mass range. Here we sketch a possible evolutionary scenario by which superChandrasekhar white dwarfs could be formed by accretion on to a commonly observed magnetized white dwarf, invoking the phenomenon of flux freezing. This opens the multiple possible evolutions ending in supernova explosions of super-Chandrasekhar white dwarfs having masses within the range stated above. We point out that our proposal has observational support, like, the recent discovery of a large number of magnetized white dwarfs by SDSS.

Subject headings: accretion, accretion disks - equation of state - novae, cataclysmic variables stars: magnetic field - supernovae: general — white dwarfs
\end{abstract}

\section{INTRODUCTION}

Identifying the progenitors of type Ia supernovae is an extremely important and ongoing research issue (Howell 2011). These supernovae are considered to be standard candles for cosmic distance measurements and hence are probes for studying the expansion history of the universe (Perlmutter et al. 1999). According to the general consensus, a carbon-oxygen white dwarf in a binary system accretes matter from a companion star and gradually approaches the Chandrasekhar mass limit of $1.44 M_{\odot}$ (Chandrasekhar 1935), $M_{\odot}$ being the mass of Sun. This further triggers a thermonuclear instability in the white dwarf, resulting in a violent and extremely energetic explosion, which we observe as a type Ia supernova. However, far less is known about the nature of the companion star (Parthasarathy et al. 2007).

Adding to the puzzle further is the recent discovery of several peculiar type Ia supernovae - SN 2006gz, SN 2007if, SN 2009dc, SN 2003fg (Scalzo et al. 2010). These supernovae are distinctly overluminous, powered by a higher than usual production of nickel, and have very low ejecta velocity compared to their standard counterparts (Howell et al. 2006). They also violate the luminositystretch relation (Phillips 1993; Goldhaber et al. 2001) which prohibits them from being categorized as standard candles. Interestingly, however, these anomalies seem to be resolved if instead of the standard theory one invokes super-Chandrasekharmass white dwarfs, with masses $2.1-2.8 M_{\odot}$, as their progenitors (Scalzo et al. 2010; Howell et al. 2006; Hicken et al. 2007; $\quad$ Yamanaka et al. 2009;

\footnotetext{
${ }^{1}$ Department of Physics, Indian Institute of Science, Bangalore 560012, India; upasana@physics.iisc.ernet.in, bm@physics.iisc.ernet.in

${ }^{2}$ Department of Astronomy \& Astrophysics, Tata Institute of Fundamental Research, Mumbai 400005, India; arrao@tifr.res.in
}

Silverman et al. 2011; $\quad$ Taubenberger et al. 2011). The leading question now is the origin and stability of these super-Chandrasekhar white dwarfs. There have been a few simulations of accreting binary white dwarfs, which include differential rotation and other parameters (Hachisu et al. 2012), that try to explain the required mass range stated above. In addition, models have been proposed involving mergers of two white dwarfs, namely the double degenerate scenario (Iben \& Tutukov 1984; Scalzo et al. 2010), and core degenerate scenario (Kashi \& Soker 2011). However, a detailed theoretical understanding seems to be lacking in them.

On a fundamentally different ground, Das \& Mukhopadhyay (2012a, 2012b) have shown that strongly magnetized white dwarfs, having central fields $\sim 10^{15}-$ $10^{17} \mathrm{G}$, are capable of having super-Chandrasekhar masses in the range $2-2.6 M_{\odot}$. The basic idea employed (Das \& Mukhopadhyay 2012a, 2012b, hereafter DM1, DM2 respectively) is that, for magnetic fields $(B)$ greater than a critical field $B_{c}=4.414 \times 10^{13} \mathrm{G}$, the effect of Landau quantization on the underlying electron degenerate matter becomes significant and as a result the density of states for the electrons changes (Lai \& Shapiro 1991). This in turn modifies the corresponding equation of state (EoS) and hence the mass-radius relation of these white dwarfs. Now the additional question is to form white dwarfs with such strong magnetic fields.

In this Letter, we sketch an evolutionary scenario by which highly magnetized white dwarfs could be formed. We concentrate on the single degenerate progenitor scenario. Starting with some reasonable assumptions about the mass and the magnetic field of a normally observed magnetic white dwarf, we demonstrate that by the mechanism of flux freezing and Landau quantization a possible evolutionary link between strongly magnetized supermassive white dwarfs and the observed peculiar type Ia 
supernovae could be established. This scenario is compatible with the discovery of several (isolated) magnetized white dwarfs by SDSS having surface fields $10^{5}-$ $10^{9} \mathrm{G}$ (Schmidt et al. 2003; Vanlandingham et al. 2005) and the fact that $25 \%$ of the observed cataclysmic variables (CVs) have surface field strengths as high as $10^{7}-10^{8} \mathrm{G}$ (Wickramasinghe \& Ferrario 2000).

This Letter is organized as follows. In $\S 2$ we describe how a magnetized, sub-Chandrasekhar, accreting white dwarf evolves into a super-Chandrasekhar white dwarf. We subsequently discuss the steps that lead to the formation of multiple evolutionary tracks in the mass-radius plane, which in turn help in explaining the observed super-Chandrasekhar (progenitor) mass range of the peculiar type Ia supernovae. In $\S 3$ we study the timescale of evolution of these magnetized accreting white dwarfs. Finally we conclude in $\S 4$ with a discussion about the observational evidence supporting our proposed evolutionary scenario.

\section{EVOLUTIONARY PATH FROM A SUB-CHANDRASEKHAR TO A SUPER-CHANDRASEKHAR WHITE DWARF}

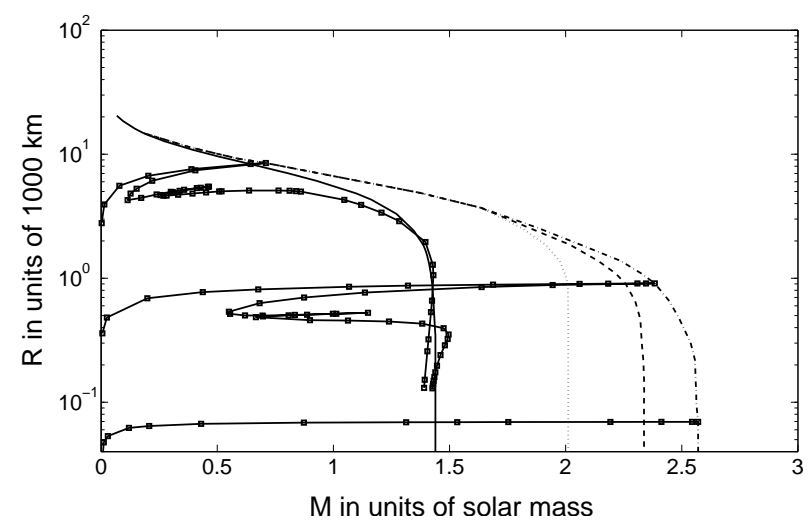

FIG. 1.- Mass-Radius relations - solid line represents Chandrasekhar's non-magnetic result; dotted, dashed and dot-dashed lines represent the evolutionary tracks of accreting magnetized white dwarfs, ending in super-Chandrasekhar type Ia supernovae with progenitor masses $2.01 M_{\odot}, 2.33 M_{\odot}$ and $2.58 M_{\odot}$ respectively The solid lines marked with squares represent from top to bottom the intermediate mass-radius relations corresponding to 50124level, 200-level and 1-level systems of Landau quantization respectively.

We consider a white dwarf having a central field several orders of magnitude higher than that of the surface, with a typical mass and radius to be $M_{0} \sim 0.2 M_{\odot}$ and $R \sim 15000 \mathrm{~km}$ respectively. Note, as justified in $\S I V . C$ of DM1, that the magnetic field might be approximately constant in a range of radii around the center of the white dwarf, which we define as internal magnetic field. This is quite a plausible assumption, since the original star collapsing to form a white dwarf might have a very large interior magnetic field compared to that observed on its surface, as has been the case for the Sun (Gough \& McIntyre 1998). Thus, it is quite likely that the values of the central and surface magnetic fluxes (in the initial star and hence in the white dwarf) would be significantly different, developing a coreenvelope boundary. Hence, we start with an internal magnetic field $B_{\text {int }} \sim 5 \times 10^{12} \mathrm{G}$ along with a surface field $B_{s}$ at least three orders of magnitude lower than $B_{\text {int }}$. Such a white dwarf still has $B<B_{c}$ and it lies on Chandrasekhar's (non-magnetic) mass-radius relation having EoS similar to that of a nonmagnetic case. Now as the white dwarf accretes matter, there is an amplification of its central magnetic field as a consequence of the increase in central density (via flux freezing theorem) due to the contraction in size of the white dwarf (Cumming 2002). This leads the central magnetic field to eventually exceed $B_{c}$. Hence, EoS of the underlying electron degenerate gas and the mass-radius relation of the underlying white dwarf modify, as shown in DM1 and DM2. As a result, the white dwarf transits from the mass-radius curve for the nonmagnetic EoS to that for a magnetic EoS. As the outward modified pressure counteracts the inward gravitational force, a quasi-equilibrium state attains, which is determined by the degree of Landau quantization of the system. Larger the magnetic field, smaller is the number of Landau levels occupied by the electrons, leading to more massive white dwarfs (DM1, DM2). Hence, as accretion continues, the massradius curve of the white dwarf deviates more and more from that of Chandrasekhar's. In Figure1, we show three mass-radius relations (solid curves marked with squares) following DM1, for magnetized white dwarfs with three different values of $B_{\text {int }}-1.7 \times 10^{13} \mathrm{G}, 4.4 \times 10^{15} \mathrm{G}$ and $8.8 \times 10^{17} \mathrm{G}$, for the top to bottom curves respectively, through which the evolving white dwarf, with initial mass, radius and magnetic fields mentioned above, passes at different intermediate stages.

Following Bandyopadhyay et al. (1997), we adopt a profile for magnetic field in our study assuming the surface and central magnetic fluxes of the initial and final white dwarfs are individually conserved as the evolution progresses. For example, for the white dwarf with $B_{\text {int }}=8.8 \times 10^{17} \mathrm{G}$ and $R=69.5 \mathrm{~km}$ (which is the maximum possible radius for the corresponding massradius relation), if we consider $B_{\text {int }}$ to be confined upto $R_{\text {int }}=R / 10$ (corresponding to a profile with parameters $\beta=0.01, \gamma=3.6$ (Bandyopadhyay et al. 1997)), then the above assumption holds true with surface and central fluxes respectively $1.46 \times 10^{27} \mathrm{G} \mathrm{cm}^{2}$ and $4.25 \times 10^{29} \mathrm{G}$ $\mathrm{cm}^{2}$. Note, however, that the extent of $B_{\text {int }}$ adjusts accordingly as the white dwarf evolves and hence $R_{\text {int }}$ need not be the same for the initial and final white dwarfs. Figure 1 shows how the an initial mass of the white dwarf could possibly evolve to 3 different final limiting masses, namely $2.01 M_{\odot}, 2.33 M_{\odot}$ and $2.58 M_{\odot}$. Once the corresponding final mass is reached, further accretion induces a runaway thermonuclear reaction, causing the white dwarf to explode at that mass, leading to a superChandrasekhar supernova. We note that, although we show only three tracks in Figure 1, any number of tracks are possible resulting in different final limiting masses $\lesssim 2.58 M_{\odot}$. In the following subsection we explain in detail how we obtain these multiple tracks.

\subsection{Multiple evolutionary tracks}

When the electrons occupy the ground Landau level, pressure increases monotonically as a function of density. This corresponds to a mass-radius relation where 
initially the mass increases with increasing radius (let us call it the first branch of the mass-radius relation). If the magnetic field is sufficiently high, then the radius becomes nearly independent of mass at higher central densities in this branch. From DM1, we note that whenever there is a transition from a lower Landau level to the next higher level, a kink appears in EoS, followed by a plateau - which is a small region where the pressure becomes nearly independent of the density (see Figure 1 of DM1). For a given magnetic field, the central density of the white dwarf corresponding to the kink in EoS arising at the transition from the ground to first Landau levels corresponds to a white dwarf having the maximum possible radius. Note that the systems having more than one occupied Landau level exhibit multiple branches in their mass-radius relations including the 'first branch' (for details see DM1). For example, the mass-radius relation of white dwarfs having central densities corresponding to the plateau of EoS shows a turning point and subsequent decrease of mass with decreasing radius (see Figure 2 of DM1).

Now, let us consider a white dwarf lying on an intermediate mass-radius relation in its early stage of evolution (e.g. the top most solid curve marked with squares in Figure 1), such that it has the maximum possible radius. As it evolves via accretion, its magnetic field and mass increases with decreasing radius, such that when the white dwarf transits to the next mass-radius relation, it still has the corresponding maximum radius satisfying the flux-freezing condition. For a sufficiently high field, the white dwarf with maximum radius also corresponds to the maximum possible mass. Thus, in Figure 1, we show that the evolution of such a white dwarf gives rise to the track corresponding to the limiting mass of $2.58 M_{\odot}$, which corresponds to the new mass limit for white dwarfs proposed by Das \& Mukhopadhyay (2013). Hence, this track demarcates a zone in the mass-radius plane, beyond the right-hand side of which no further track is possible.

From the bottom two mass-radius relations (representing further intermediate masses and radii of the evolving white dwarf) in Figure 1, we notice that the evolving white dwarf passing through them may lie anywhere in a zone where the radius is independent of mass satisfying the flux freezing condition. This zone is a part of the "first branch' of the corresponding mass-radius relation. This happens at a sufficiently high field $\gtrsim 1.7 \times 10^{14} \mathrm{G}$ (which corresponds to a 5000-Landau-level system), when a part of EoS can be described by a polytropic relation of the form

$$
P=K \rho^{\Gamma}, \text { when } \Gamma=2,
$$

where $P$ and $\rho$ are respectively the pressure and density of the electron gas, $\Gamma$ is the polytropic index and $K$ is a constant depending on the magnetic field. Now on solving the magnetostatic equilibrium condition by LaneEmden formalism, one obtains the following scaling laws (DM1) for radius $(R)$ and mass $(M)$ :

$$
R \propto \rho_{c}^{(\Gamma-2) / 2}
$$

and

$$
M \propto \rho_{c}^{(3 \Gamma-4) / 2},
$$

where $\rho_{c}$ is the central density of the white dwarf. Thus when $\Gamma=2$, the radius becomes independent of the central density, while the mass becomes proportional to the central density. This indicates that the same magnetic field leads to a white dwarf having different possible masses with the same radius. Hence once the above polytropic relation starts holding true, the flux freezing condition opens the multiple possible evolutionary tracks.

\section{TIMESCALE OF MASS EVOLUTION}

Here we explore the typical timescale of evolution of the accreting magnetic white dwarf systems with assumed parameters quite close to the class of magnetic CVs called the Intermediate Polars (IPs).

\subsection{Constant mass accretion rate}

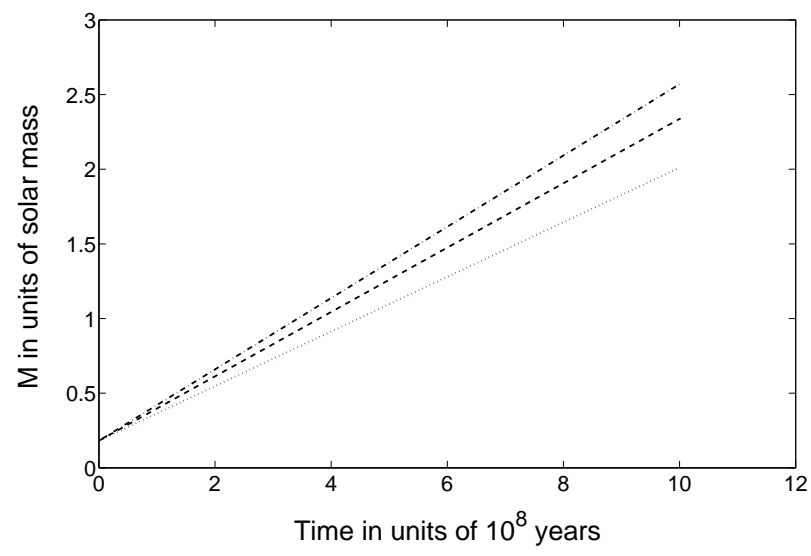

FIG. 2.- Mass evolution of accreting magnetized white dwarfs - dot-dashed, dashed and dotted lines represent constant accretion rates of respectively $2.38 \times 10^{-9} M_{\odot} \mathrm{yr}^{-1}, 2.15 \times 10^{-9} M_{\odot} \mathrm{yr}^{-1}$ and $1.83 \times 10^{-9} M_{\odot} \mathrm{yr}^{-1}$ for supernova explosions occurring at the same time, for progenitor masses $2.58 M_{\odot}, 2.33 M_{\odot}$ and $2.01 M_{\odot}$ respectively.

Figure 2 shows the evolution of an initial white dwarf of mass $0.2 M_{\odot}$ and radius $15000 \mathrm{~km}$ into the 3 superChandrasekhar white dwarfs with $M \geq 2 M_{\odot}$ corresponding to Figure 1. Here we assume a constant mass accretion rate $(\dot{M})$, represented by the constant slopes of the 3 lines in Figure 2, for each case, such that they explode at the same time. The values of $\dot{M}$ (typical of IPs) are $2.38 \times 10^{-9} M_{\odot} \mathrm{yr}^{-1}, 2.15 \times 10^{-9} M_{\odot} \mathrm{yr}^{-1}$ and $1.83 \times 10^{-9} M_{\odot} \mathrm{yr}^{-1}$ for the exploding masses of $2.58 M_{\odot}$, $2.33 M_{\odot}$ and $2.01 M_{\odot}$ respectively. We see that the system with a higher $\dot{M}$ accumulating more mass in the same time (roughly a billion year) gives rise to a more massive supernova explosion.

Figure 3(a) shows that how the exploding mass of $2.33 M_{\odot}$ is attained by 3 different $\dot{M} \mathrm{~s}-3 \times 10^{-9} M_{\odot} \mathrm{yr}^{-1}$, $2.5 \times 10^{-9} M_{\odot \mathrm{yr}^{-1}}$ and $2 \times 10^{-9} M_{\odot \mathrm{yr}^{-1}}$ - resulting in the explosions occurring at different times.

\subsection{Varying mass accretion rate}

The most favored models for type Ia supernova progenitors invoke a very high $\dot{M} \gtrsim 10^{-7} M_{\odot} \mathrm{yr}^{-1}$, such that the accreted hydrogen and helium can burn steadily (Cumming 2002). These values of $\dot{M}$ are observed in supersoft X-ray sources and symbiotic binaries 


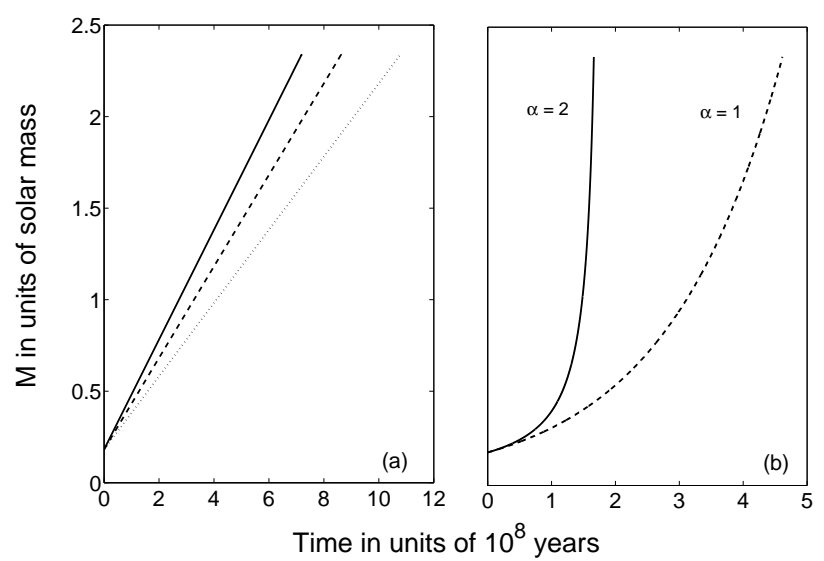

FIG. 3.- Mass evolution of accreting magnetized white dwarf with (a) constant accretion rate - solid, dashed and dotted lines represent $3 \times 10^{-9} M_{\odot \mathrm{yr}^{-1}}, 2.5 \times 10^{-9} M_{\odot \mathrm{yr}^{-1}}$ and $2 \times$ $10^{-9} M_{\odot} \mathrm{yr}^{-1}$ respectively, (b) varying accretion rate - solid and dashed lines correspond to $\alpha=2$ and $\alpha=1$ respectively.

(Cumming 2002; Parthasarathy et al. 2007). At lower

$\dot{M}$ s, hydrogen burning is unstable and occurs in flashes, while at higher rates an extended envelope is formed (van den Heuvel et al. 1992).

We now plan to invoke a range of $\dot{M}$ s, low to high, in a single model evolution of the white dwarf and hence consider

$$
\dot{M}=\left(\frac{M}{M_{0}}\right)^{\alpha} \times 10^{-9} \mathrm{yr}^{-1},
$$

where $\alpha$ is a parameter that determines the functional dependence of $\dot{M}$ on the instantaneous mass $\left(M \geq M_{0}\right)$ of the white dwarf.

In Figure 3(b) we show two cases corresponding to $\alpha=1$ and $\alpha=2$, having the same final exploding mass, namely $2.33 M_{\odot}$. In the case with $\alpha=1, \dot{M}$ varies from $10^{-9} M_{\odot} \mathrm{yr}^{-1}$ to $1.3 \times 10^{-8} M_{\odot \mathrm{yr}^{-1}}$. The evolution is slow in this case and the supernova explosion occurs in $\sim 5 \times 10^{8}$ years. In the case with $\alpha=2, \dot{M}$ varies from $10^{-9} M_{\odot} \mathrm{yr}^{-1}$ to $1.7 \times 10^{-7} M_{\odot \mathrm{yr}^{-1}}$. The evolution here is more than twice as fast as the previous case and the supernova explosion occurs in $\sim 2 \times 10^{8}$ years. Hence, $\alpha \geq 2$ is supposed to validate the underlying white dwarfs as progenitors of the observed peculiar type Ia supernovae.

\section{DISCUSSION AND CONCLUSIONS}

Based on the theoretical work on strongly magnetized white dwarfs having super-Chandrasekhar masses (DM1, DM2), we have discussed possible evolutionary paths that lead from magnetized accreting white dwarf binaries to the peculiar type Ia supernova explosions. Multiple evolutionary tracks are possible in the mass-radius plane, which covers a mass range $\sim 2-2.6 M_{\odot}$, that lies within the observational limits. We have considered both constant as well as varying $M_{\odot}$, in order to estimate the timescale of occurrence of the supernova events.

Highly magnetized white dwarfs have been discovered by SDSS having surface fields in the range $10^{5}-$ $10^{9} \mathrm{G}$ (Schmidt et al. 2003; Vanlandingham et al. 2005; Wickramasinghe \& Ferrario 2000). Note that their central fields are expected to be a few orders of magnitude higher. The properties that we have assumed in our computation are similar to those seen in IPs. They have surface magnetic fields ranging $10^{5}-10^{7} \mathrm{G}$ and they are high accretors with $\dot{M} \approx(0.2-4) \times 10^{-9} M_{\odot} \mathrm{yr}^{-1}$ (Warner 1995). Cumming (2002) studied the evolution of magnetic fields in accreting white dwarfs and found that surface magnetic fields are reduced significantly for $\dot{M}>\dot{M}_{c} \approx(1-5) \times 10^{-10} M_{\odot} \mathrm{yr}^{-1}$, due to the advection of the field into the interior of the white dwarf by the accretion flow. This presumably increases the central magnetic field (Cumming 2002). Further, certain dwarf novae (a subclass of CVs) like RU Peg, are expected to show short and long term variations in $\dot{M} \mathrm{~s}$, ranging from as low as $(1-2) \times 10^{-9} M_{\odot \mathrm{yr}^{-1}}$ to as high as $10^{-7} M_{\odot \mathrm{yr}^{-1}}$ (Parthasarathy et al. 2007). Hence, it is quite conceivable that IPs with high internal field and low surface field with an increasing $\dot{M}$ can lead to superChandrasekhar white dwarfs causing the peculiar supernova Ia explosions. Moreover, for $\alpha=2$ according to our chosen model, the desired $\dot{M}$ for a type Ia supernova explosion to occur is attained starting from an initial, lower rate, typically observed in IPs. Note that peculiar type Ia supernovae arise, according to our argument, from highly magnetized CVs, which are presumably $10 \%$ of all magnetized CVs. Hence about $2.5 \%$ of all CVs should lead to super-Chandrasekhar objects, which is consistent with the observed rate $(1-2 \%)$ of peculiar type Ia supernovae (Scalzo et al. 2012).

Apart from the advection of the magnetic field into the interior caused by accretion, there are other ways of generating such strong fields. ${ }^{3}$ Interestingly, recent observations of magnetic Ap and Bp stars indicate that they have a magnetic flux in the range $10^{26}-10^{27} \mathrm{G} \mathrm{cm}^{2}$, which is very similar to that observed in magnetized white dwarfs (Ferrario \& Wickramasinghe 2005). This strongly points towards the fact that the magnetic fields of highly magnetized white dwarfs are fossil remnants from their mainsequence progenitor stars (Tout et al. 2004). Simulations of magnetic field evolution in Ap stars strongly support the idea of this field itself being a fossil remnant from the interstellar medium, generated during the process of star formation (Braithwaite \& Spruit 2004). Results of these simulations to hold true, the new born star is required to have an initial field mainly confined to the core, which can be justified from the flux freezing theory during its formation (Braithwaite \& Nordlund 2006). Moreover, the contraction of a typical interstellar cloud of radius $\sim 0.1 \mathrm{pc}$, mass $\sim M_{\odot}$ and having a frozen-in magnetic field $\sim 3 \times 10^{-6} \mathrm{G}$ could give rise to a field $\sim 10^{8}$ $\mathrm{G}$ in the resulting star (Shapiro \& Teukolsky 1983) with solar radius. Thus, a star with such a high centrally concentrated field is a plausible progenitor of the strongly magnetized super-Chandrasekhar white dwarfs discussed in this Letter. These stars also have convective cores and in some special cases a dynamo mechanism might occur to generate a high magnetic field therein, leading to a (central) magnetic flux as high as $\sim 10^{30} \mathrm{G} \mathrm{cm}^{2}$, similar to that of the magnetized white dwarfs mentioned in $\S 2$. The incidence of such main-sequence magnetic stars might be rare, but so are the super-Chandrasekhar

3 However, see the Appendix of DM1, which discusses that even a relatively weaker field than that considered here can give rise to the super-Chandrasekhar masses. 
supernova events.

If the single-degenerate accreting scenario is the correct progenitor model for the overluminous (peculiar) type Ia supernovae, then observationally such high magnetic, high mass white dwarfs with small radius (also with high $\dot{M})$ could be one of the plausible progenitors of these supernovae. However, a detailed investigation needs to be carried out in order to understand the physics of explosions of these white dwarfs and if they indeed produce the light curves similar to that of the peculiar type Ia supernovae.

Now the white dwarfs would be seen as peculiar, superChandrasekhar objects only during a small fraction of their life time, which is $\sim 0.05$ for $\alpha=2$. Hence, about $\sim 0.12 \%$ of the CVs with varying $\dot{M}$ would be likely candidates for peculiar objects. The varying $\dot{M}$ is further justified by astronomical indications, which imply higher accretion rates at later times of the evolution of magnetized massive white dwarfs (Wang \& Han 2012; Toonen et al. 2013; Nomoto et al. 2007). We speculate that these white dwarfs will have X-ray luminosity in between commonly observed accreting white dwarfs and accreting neutron stars (in the range of $10^{35}-$ $10^{38}$ ergs s$^{-1}$ ). Bright X-ray selected CVs (and other objects) might harbor some of these peculiar superChandrasekhar objects.

This work is partly supported by ISRO project Grant No. ISRO/RES/2/367/10-11. U.D. thanks CSIR, India for financial support. The authors would like to thank Arnab Rai Choudhuri of IISc for discussion.

\section{REFERENCES}

Bandyopadhyay, D., Chakrabarty, S., \& Pal, S. 1997, Phys. Rev. Lett., 79, 2176.

Braithwaite, J., \& Spruit, H. C. 2004, Nature, 431, 819.

Braithwaite, J., \& Nordlund, A. 2006, A\&A, 450, 1077.

Chandrasekhar, S. 1935, MNRAS, 95, 207.

Chanmugam, G., Ray, A., \& Singh, K. P. 1991, ApJ, 375, 600.

Cumming, A. 2002, MNRAS, 333, 589.

Das, U., \& Mukhopadhyay, B. 2012a, Phys. Rev. D, 86, 042001; DM1.

- 2012b, Int. J. Mod. Phys. D, 21, 1242001; DM2.

Das, U., \& Mukhopadhyay, B. 2013, Phys. Rev. Lett., 110, 071102.

Ferrario, L., \& Wickramasinghe, D. T. 2005, MNRAS, 356, 615.

Goldhaber, G., et al. 2001, ApJ, 558, 359.

Gough, D. O., \& McIntyre, M. E. 1998, Nature, 394, 755.

Hachisu, I., Kato, M., Saio, H., \& Nomoto, K. 2012, ApJ, 744, 69.

Hicken, M., et al. 2007, ApJ, 669, L17.

Howell, D. A. 2011, Nature Comm., 2, 350.

Howell, D. A., et al. 2006, Nature, 443, 308.

Iben, I., \& Tutukov, A. V. 1984, ApJS, 54, 335.

Kashi, A., \& Soker, N. 2011, MNRAS, 417, 1466.

Lai, D., \& Shapiro, S. L. 1991, ApJ, 383, 745.

Nomoto, K., Saio, H., Kato, M., \& Hachisu, I. 2007, ApJ, 663, 1269.

Parthasarathy, M., Branch, D., Jeffery, D. J., \& Baron, E. 2007, New Ast. Rev., 51, 524.
Perlmutter, S., et al. 1999, ApJ, 517, 565.

Phillips, M. M. 1993, ApJ, 413, L105.

Scalzo, R. A., et al. 2010, ApJ, 713, 1073.

Scalzo, R. A., et al. 2012, ApJ, 757, 12.

Schmidt, G. D., et al. 2003, ApJ, 595, 1101.

Shapiro, S. L., \& Teukolsky, S. A. 1983, Black Holes, White Dwarfs and Neutron Stars: The physics of compact objects (John Willey \& Sons, New York).

Silverman, J. M., et al. 2011, MNRAS, 410, 585.

Taubenberger, S., et al. 2011, MNRAS, 412, 2735.

Toonen, S., Nelemans, G., Bours, M., \& Portegies Zwart, S. arXiv:1302.0837.

Tout, C. A., Wickramasinghe, D. T., \& Ferrario, L. 2004, MNRAS, 355, L13.

van den Heuvel, E. P. J., Bhattacharya, D., Nomoto, K., \& Rappaport, S. A. 1992, A\&A, 262, 97.

Vanlandingham, K. M., et al. 2005, AJ, 130, 734.

Wang, B., \& Han, Z. 2012, New Ast. Rev., 56, 122

Warner, B. 1995, Cataclysmic Variable Stars (Cambridge: Cambridge Univ. Press)

Wickramasinghe, D. T., \& Ferrario, L. 2000, Pub. Ast. Soc. Pac., $112,873$.

Yamanaka, M., et al. 2009, ApJ, 707, L118. 